\title{
Verbs in space: Axis and direction of motion norms for 299 English verbs
}

\author{
LOTTE METEYARD \\ University College London, London, England \\ and MRC Cognition and Brain Science Unit, Cambridge, England \\ AND \\ Gabriella Vigliocco \\ University College London, London, England
}

\begin{abstract}
A strong body of work has explored the interaction between visual perception and language comprehension; for example, recent studies exploring predictions from embodied cognition have focused particularly on the common representation of sensory-motor and semantic information. Motivated by this background, we provide a set of norms for the axis and direction of motion implied in 299 English verbs, collected from approximately 100 native speakers of British English. Until now, there have been no freely available norms of this kind for a large set of verbs that can be used in any area of language research investigating the semantic representation of motion. We have used these norms to investigate the interaction between language comprehension and low-level visual processes involved in motion perception, validating the norming procedure's ability to capture the motion content of individual verbs. Supplemental materials for this study may be downloaded from brm.psychonomic -journals.org/content/supplemental.
\end{abstract}

A central question in psycholinguistic and cognitive science research concerns how semantic information can refer to things in the world and therefore serve language in making words and sentences meaningful. Wider debates about the nature of cognitive representation have repeatedly focused on one question: Being grounded in our embodied experience, is information about the world embedded within perceptual and motor activity or is this information transformed into a qualitatively different, symbolic format? These two traditional views, embodied and symbolic, have existed in various guises within various debates over such topics as mental imagery (Kosslyn, 1996; Pylyshyn, 1985), conceptual representation ("nonlinguistic psychological representation[s] of a class of entities in the world"-Murphy, 2002, p. 385; see also Barsalou, 1999), and semantic representations (the meaningful content of verbal labels; Fodor, 1987; Lakoff, 1987). The symbolic tradition starts with the cognitive domain, what is "in our heads," whereas, the embodied tradition starts with the experiential domain, what is "in our world." According to embodied theories, sensory and motor systems are recruited (i.e., directly and necessarily activated) to represent, at a minimum, the semantic content of words that refer to objects or events experienced through the sensory and motor systems. For example, such words as kick and lick recruit motor systems involved in kicking and licking (Pulvermüller, 2001), and such words as rise and fall recruit sensory systems involved in motion processing (Meteyard, Bahrami, \& Vigliocco, 2007; Meteyard, Zokaei, Bahrami, \& Vigliocco, 2008).

The norms presented here were collected in order to support investigations into embodied theories of semantic representation. We were interested in the lexical representation of verbs (events) rather than nouns (objects) and focused on motion events as a promising area for exploring the links that connect language, perception, and action that are at the core of embodied theories (Bergen, Lindsay, Matlock, \& Narayanan, 2007; Kaschak et al., 2005; Kaschak, Zwaan, Aveyard, \& Yaxley, 2006; Meteyard et al., 2007; Meteyard et al., 2008; Richardson, Spivey, Barsalou, \& McRae, 2003). We embarked on a large-scale norming procedure for a large number of verbs that were selected for their potential motion content. Researchers can select items from this set on the strength of their normed scores for a particular axis or direction of motion, rather than relying on intuitive judgments that might be open to confirmation bias. Our aim was to establish sets of verbs with consistent semantic properties that could be used in online behavioral experiments and to have a set large enough that control items (with no particular direction of motion) could also be selected. Norming a large number of verbs also gave us greater scope for future use. To our knowledge, there are no freely available norms that can be used to select items on the basis of their motion 
content. Therefore, these norms are useful most obviously for investigating interactions between visual processing (axis and direction of motion) and comprehension; the basis of the norming procedure used here owes its origins to such experiments.

\section{Language Comprehension and Visual Perception}

A growing body of work explores interactions between comprehension and visuospatial processing, with experimental sentences that describe motion that is upward, downward, toward the observer, or away from the observer. Richardson, Spivey, Edelman, and Naples (2001) gathered ratings for 30 verbs, using these norms to class items as horizontal (e.g., pull, point, want, offend) and vertical (e.g., sink, float, hope, respect). Sentences were created from these verbs. Concrete sentences referred to actual objects moving (e.g., The ship sinks in the ocean), whereas abstract sentences referred to emotional states or abstract quantities (e.g., The store owner increases the price and The girl hopes for a pony). In one experiment, the sentences were presented aurally, while pictures displayed synchronously depicted the actors in the sentence. For example, the sentence The balloon floats through the window was accompanied by a picture of a balloon when the word balloon was heard, and so on. During sentence comprehension, pictures were presented centrally. In a test phase, two pictures were presented in either a horizontal or vertical arrangement and participants had to judge whether those pictures had been presented together during comprehension. The concrete and abstract sentences were analyzed together, and faster reaction times were observed for vertically arranged pictures for the "vertical" sentences, although the horizontal sentences did not show comparative effects (Richardson et al., 2003, Experiment 2). Post hoc analyses showed that the effect was significant for concrete, but not for abstract, sentences. Another experiment showed that presenting these sentences binaurally affected the speed of categorization for a shape that appeared in the upper, lower, left, or right visual field. Categorization was slower when the shape location (vertical or horizontal) was congruent with the sentence than when it was incongruent; this difference was stronger for abstract sentences. These experiments demonstrated that sentence comprehension may activate embodied "image schemata" that facilitate memory processes (Richardson et al., 2003, Experiment 2) or interfere with visuospatial attention (Richardson et al., 2003, Experiment 1). Bergen et al. (2007) used this shape categorization task and presented shapes in the upper and lower visual fields, preceded by sentences that described upward (e.g., The mule climbed) or downward (e.g., The chair toppled) motion. Concrete sentences with verbs referring to intransitive motion were found to produce interference (Experiment 1), as were sentences with concrete nouns (e.g., The ceiling cracked and The cellar flooded; Experiment 2). Bergen et al. discussed their results in terms of the image schema or visual imagery that the sentence evokes (in line with Perky, 1910; cf. Estes, Verges, \& Barsalou, 2008). The results are taken to support embodied theories of semantics, because the image schemata are thought to have visuospatial content that would recruit perceptual systems involved in visual and spatial processing.

Effects have also been found for static pictures that approximate motion (Zwaan, Madden, Yaxley, \& Aveyard, 2004). On each trial, a sentence was followed by two pictures, separated from each other by a brief mask; participants judged whether the pictures were the same. On critical trials, the sentences described the egocentric motion of a ball toward or away from the comprehender (e.g., The pitcher hurled the softball at you and You hurled the softball at the pitcher). The pictures were of a ball similar to that described in the sentence (e.g., for the above sentences, a softball), with the second image slightly smaller or slightly larger than the first to approximate the motion of the ball toward (larger) or away (smaller). When the motion of the ball in the sentence was congruent with the pictured motion, judgments were faster. This result replicated a congruency advantage seen in other experiments. Here, sentence comprehension was followed by the categorization or naming of a picture that was congruent or incongruent with the visual properties (e.g., shape, orientation) of objects described in the sentences (e.g., The pencil is in the cup, followed by a picture of a vertical [congruent] or horizontal [incongruent] pencil) (e.g., Stanfield \& Zwaan, 2001; Zwaan, Stanfield, \& Yaxley, 2002).

In a study that used video of actual motion rather than a spatial or visual approximation, Kaschak et al. (2005) presented sentences aurally for grammaticality or sensibility judgments, while black-and-white graphics were displayed on a screen. The sentences described vertical or egocentric motion (e.g., upward, The smoke rose into the sky; away from the body, You backed out of the driveway), and the graphics depicted motion in two planes (upward or downward vertical motion and motion toward or away from the observer). Thus, sentences were either congruent or incongruent with the visual motion. Both grammaticality and sensibility judgments were slower when the visual display was congruent with the motion in the sentence (see Kaschak et al., 2006, for an extension of this original experiment). The authors discussed the results in terms of the competition for resources. Specifically, they inferred that the motion sentences directly activated visual motion processing areas. When this semantic motion was in the same direction as the visual motion display, the two inputs competed for the same visual areas that process a particular direction of motion; this competition slowed judgments of the sentences.

\section{Eye Movements}

Eye movements are used increasingly in investigations of language comprehension (see, e.g., Henderson \& Ferreira, 2004). Eye movements provide a measure of where attention is directed and, therefore, can be used to determine the immediate object on which cognitive processes are focused: You can move attention without moving the eyes, but you cannot move the eyes without moving attention. The duration and the location of participants' fixations (where they are looking) can be measured with millisecond accuracy, and two dependent variables are typically extracted: the time spent fixating during a trial 
and the course of eye movements across a whole trial. Eye movements can be used to track the integration of linguistic stimuli and physical experience, providing evidence that participants map words to their referent objects quickly and in accordance with syntactic constraints and the use of particular prepositions (Chambers, Tanenhaus, Eberhard, Filip, \& Carlson, 2002; Chambers, Tanenhaus, $\&$ Magnuson, 2004). Other studies have used this principle to explore the qualities that make distractors compete effectively with a target object (Dahan \& Tanenhaus, 2005; Myung, Blumstein, \& Sedivy, 2005), supporting the embodied idea that perceptual information is implicated in semantic representation.

Laeng and Teodorescu (2002) demonstrated that eye movements during observation of a visual display were closely correlated with eye movements made when participants were asked to imagine the observed display while looking at a blank screen. Participants who were asked to fixate in one place when the visual display was presented also kept their eyes fixated when they imagined the display. This supports the embodied prediction that sensory and motor systems are involved in imagination, since it provides evidence that mental imagery reactivates eye movements made during experience (observation of the display). Spivey and Geng (2001, Experiment 2) corroborated this finding by showing that saccades are made to the location of previously seen objects during explicit recall of the objects; but they also presented participants with auditory scene descriptions that referred to objects and events extending upward, downward, leftward, or rightward. Each story began with "Imagine ..." and then described a scene illustrative of a particular motion - for example, a downward scene with the listener on top of a cliff watching people rappel down the cliff face. Significantly more eye movements were congruent with the implied direction of the scene as compared with that same direction for a control story with no directional bias (Spivey \& Geng, 2001, Experiment 1). This result has been replicated when participants were not instructed to imagine the scene and had their eyes closed. (Eye movements were inferred from the way the corneal bulge shaped the eyelid and reflected a luminant spot.) There were more saccades in the implied direction of a scene description than in the unimplied directions (Spivey, Richardson, Tyler, \& Young, 2000). In concordance with these findings, eye movements have also been shown to reflect fictive motion as well as the literal location of objects and events. Matlock and Richardson (2004) presented participants with simple pictures that depicted one object extended horizontally and another extended vertically (e.g., a shelf and a cord). While they were looking at the pictures, participants listened to fictive or no-motion sentences (e.g., The cord runs along the wall vs. The cord is on the wall). Participants spent significantly more time looking at the relevant object when the sentence contained fictive motion, because, the authors argued, fictive motion is represented dynamically via simulation of real motion and because eye movements reflect this dynamic representation. Together, this evidence suggests that eye movements may be integral to visual imagery and sentence comprehension (when the sentence describes motion in particular directions or the location of objects), which would support the embodied prediction that visual systems are necessarily involved in processing imaginative or semantic content that has visual referents.

As the above summary shows, a growing body of evidence supports the idea of an interaction between language comprehension and visual perception. However, at present, experimenters judge the directional content of linguistic items either intuitively (e.g., Stanfield \& Zwaan, 2001) or by completing small-scale norming (Richardson et al., 2001). The norms presented in the present article were collected to support work that explored the interaction between the semantic content of words that refer to motion and the perception of visual motion. We hope that our making these norms available will enable researchers to extract both experimental (directional) items as well as control items (without salient directionality) from the same database, for use in such experiments.

\section{Method}

One previous study used a norming procedure to demonstrate the axis and direction of motion for concrete and abstract verbs (Richardson et al., 2001). We adapted this procedure, in which norming was completed using a forced choice task, with verbs placed within rebus sentences, in which shapes serve as sentence subjects and objects, with a written verb between each pair of shapes.

We adapted this forced-choice task so that the sentences presented for judgment took the form " "circle' verbs "square"”; it was decided to use words, and not rebus sentences as in Richardson et al. (2001), to mitigate a possible stronger visual bias in picture selection. Participants may have been more likely to select a picture with a circle on the left and a square on the right having seen this visual arrangement in the rebus sentence. We felt that this would be less likely if pictures were replaced with words, so that the rebus sentence would not visually map onto the options directly. The pictures presented for selection balanced left and right positioning of the circle (subject) and square (object), so some spatial bias may be unavoidable: The rebus sentences always have the circle leftmost, the typical position of the subject within a sentence, and the square rightmost, the typical position of the object. There is evidence that temporal markers make information more or less available in narrative comprehension (Zwaan, 1996). Participants read narratives that contained adverbials, such as "a moment/hour/day later," and then responded to probe items, which were words taken from the narrative section preceding the adverbial. Participants took longer to respond to the probe when it was followed by "a day later" than when it was followed by "a moment later," which suggested that the adverbial time shift had made the probe word less salient (Zwaan, 1996). We assumed that similar processes may occur during the comprehension of simple sentences, so we used the present tense to make the activity involved in the verb more salient. We did not want to use the present progressive form "'circle' is Xing 'square'," because this introduced an extra item (the auxiliary be) into the sentence. Along with avoiding the use of the auxiliary, the simple present form " circle' Xs 'square" also implies habitual behavior, which we felt foregrounded the event further. Verbs that would require a preposition to accommodate an object were presented in intransitive form (e.g., " "circle' runs" rather than "“circle' runs to 'square"). This was done because prepositions such as to and at communicate considerable spatial information that would be confounded with the information given in the verb alone.

Participants had to choose from among several options but were allowed to express an order of preference. Although we planned to select experimental items suggesting strong upward or downward motion, the norming was carried out on a large set of items, some of which may strongly suggest directional motion in more than one direction (e.g., expand, swell, bounce) or in no particular direction 
(e.g., eat, kick, attack). By letting participants choose many (or all) of the pictures, we hoped to capture these consistencies (e.g., upward and downward motion for hop and bounce) or inconsistencies (when no direction is preferred across participants, more randomly ordered selection of multiple pictures should be produced). Providing a limited set of pictures constrained responses to the motion axes of interest (horizontal and vertical). However, rather than limiting their choice to one or two pictures, we established preference by allowing participants both to choose the number of pictures and to rank them by preference. We hoped that this approach would constrain choices by the directional content of the items rather than forcing a choice of only one or two pictures per item.

In cases where no picture was preferred, participants would select more pictures with no particular order of preference; whereas, if one was strongly preferred, it would be the only one selected and would have the highest order of preference. Sixteen pictures were generated to represent all possible combinations of the pictured elements for transitive and intransitive events: 8 corresponded to transitive events (a circle and a square), and 8 corresponded to intransitive events (only a circle). The pictures incorporated two axes (vertical and horizontal) and two directions in which the event could occur (toward and away from the subject/circle). The pictures also balanced the positioning of the circle and square (left, right, top, bottom). For each sentence, 8 pictures were presented for the participant to choose from. Transitive sentences were presented with "transitive" pictures, and intransitive sentences were presented with "intransitive" pictures. The pictures were presented in two rows of 4 , and each row had pictures with either a vertical or horizontal axis. To control for order effects, there were two possible orders of presentation for the pictures within each row. In the top left-hand corner of each picture was a small box, which the participants were instructed to use for marking their choice (see Figure 1 and the online materials for pdf documents with complete instructions and norming materials).

A list of 278 verbs was compiled, taken mostly from Levin (1993). Verbs were selected for their probable spatial qualities (e.g., verbs of inherent direction, such as to fall or to rise, and calibratable changeof-state verbs, in which a positive or negative change of state occurs in a measurable way, such as to climb or to decrease). Possible control items were also selected that had no probable spatial qualities (e.g., to finish, to know, to laugh). It was decided that 17 of the verbs would be judged in both their transitive and intransitive forms, with the final score an average of both (these are marked in the online materials with an asterisk in the "list" column). This raised the total number of verbs to be normed to 295 . These 17 verbs were equally usable in a transitive or intransitive form (e.g., to decrease, to move), and it was therefore necessary to have both possibilities realized in

\begin{tabular}{|lll|}
\hline 'circle' & writes & 'square' \\
\hline
\end{tabular}
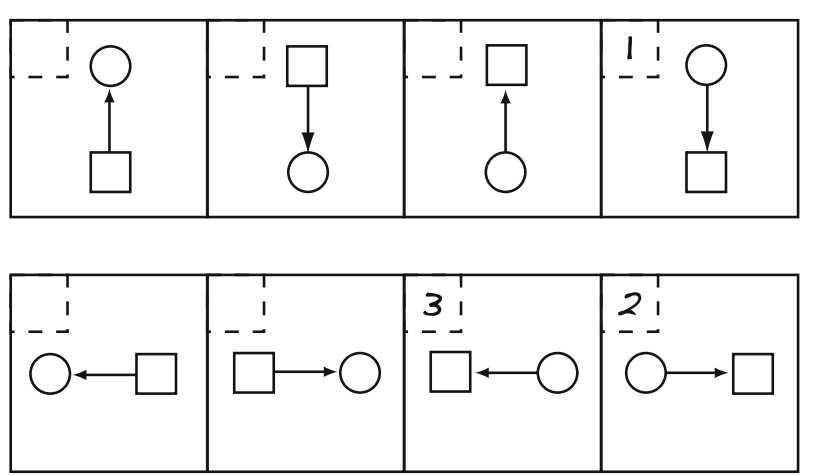

Figure 1. Example of rebus sentence and picture choices used in norming procedure. the norming, so that future use would not be constrained (in the online materials, a separate file is provided with these verbs and their transitive/intransitive scores. In the main file, their averaged score is given). The verbs were then divided quasi-randomly into three lists (two of 98 verbs and one of 99 verbs) so that each verb appeared only once per list. To identify the list in which the item appeared, a 1,2, or 3 is marked in the "list" column in the online materials. We felt that these smaller lists would be more manageable for participants to norm.

Following this initial large-scale norming, an additional 21 verbs were normed using the same procedure, except that electronic rather than paper booklets were constructed. These additional verbs were added to the original set, bringing the total to 299 . These are marked in the online materials with the word add in the "list" column.

Participants. A total of 96 native English speakers participated in norming the set of 295 verbs; 32 rated each list. Two participants had to be excluded for mistakenly filling out the booklets. Fifteen native English speakers participated in norming the additional 21 verbs.

The only condition for recruiting participants was that they were native English speakers (speaking/learning English from birth and currently using English as their primary or first language). Culturally, the participant sample was Western/European, because the vast majority were undergraduate students attending University College London and were therefore predominantly British or American.

Apparatus. For the set of 295 verbs, A5 booklets were created for each participant. On each page, a verb was placed in a sentence (outlined above), beneath which were eight pictures. Participants used pencils or pens to indicate their choices of pictures.

For the additional 21 verbs, Excel workbooks were constructed with one rebus sentence per row in an Excel worksheet, from the second row onward of the leftmost column (column A). Eight pictures were placed in the top row of columns B-I, with one worksheet for transitive events and one for intransitive events. Participants filled in their choices by typing numbers in the relevant cells of the second row, below their chosen picture.

Design. The design was within subjects: For the set of 295 verbs, each participant normed one third of the total set of verbs; for the additional verbs, participants normed all 21 verbs. The order of verbs in the booklet (or Excel workbook) was randomized for each participant. The independent variable was the verb being judged, and the dependent variables were the consistent elements across the choice of pictures (axis of motion, direction of motion, motion toward or away from the circle/square).

Procedure. The participants were provided with an instruction sheet that described the stimuli they were going to see. They were instructed to choose at least one picture for each sentence and as many as they felt were applicable for the event that the sentence described. Participants were also instructed to rank their choices in order of preference, with 1 being the most preferred, 2 the next most preferred, and so on. Order of preference was enforced in order to allow assessment of agreement across participants. Participants were told that it was a subjective judgment task; that, therefore, there were no right or wrong responses; and that, as such, responses should be made on the basis of whatever they felt was most appropriate. As soon as it was clear that participants understood the nature of the task, they were provided with the booklets (see Figure 1 for an example; full instructions are provided as part of the online materials).

\section{Analysis}

The choices were scored by weighting the first choices (as established by order of preference) more heavily than the last ones. Points were allocated to each picture on the basis of how many pictures each participant had chosen overall and the order of preference given to each choice. Points were allocated in descending magnitude in the order of preference. For the scoring system, we considered only the ranking of choices (so, the number of points allocated 
Table 1

Scoring System Used for Picture Choices

\begin{tabular}{ccccccccc}
\hline \multirow{2}{*}{$\begin{array}{c}\text { Number } \\
\text { of Picture }\end{array}$} & \multicolumn{7}{c}{$\begin{array}{c}\text { Order of Preference As } \\
\text { Chosen by Participant }\end{array}$} \\
\cline { 2 - 9 } Choices & 1 & 2 & 3 & 4 & 5 & 6 & 7 & 8 \\
\hline $1-4$ & 4 & 3 & 2 & 1 & & & & \\
5 & 4 & 4 & 3 & 2 & 1 & & & \\
6 & 4 & 4 & 3 & 3 & 2 & 1 & & \\
7 & 4 & 4 & 3 & 3 & 2 & 2 & 1 & \\
8 & 4 & 4 & 3 & 3 & 2 & 2 & 1 & 1 \\
\hline
\end{tabular}

Note-The cell values are the points allocated to each picture choice in the scoring procedure.

is essentially arbitrary). We used the following reasoning: For each sentence, there was a minimum choice of one picture and a maximum choice of eight. Initial surveys of the booklets showed that most participants chose somewhere between one and four pictures; for this reason, the scoring system was based around four choices and was modified for instances in which there were more than four. Thus, for choices of from one to four pictures, the first choice was given 4 points and the rest were scored in descending magnitude (e.g., the fourth choice received 1 point). For cases in which more than four pictures were chosen, the initially preferred pictures were scored more evenly. See Table 1 for the scoring system.

According to this scoring system, the more pictures that were chosen for a sentence, the more scores were distributed equally across the chosen pictures. For example, when eight pictures were chosen, choices were scored as pairs (1 and 2, 3 and 4, etc.) that received an equal number of points. Although it may have been simpler to allocate points strictly according to order of preference (i.e., first choice always has 8 points, second choice always has 7 , and so on), we wanted to capture the fact that the choice of more pictures reflected less conviction about which best captured the motion in the verb. It is logical to assume that, the fewer pictures that were chosen, the more relevant and important were the attributes of the chosen pictures and the higher weighting they should receive. In contrast, the more pictures that were chosen, the less relevant were the attributes of any one picture, so the weighting should be distributed more evenly. Our scoring system achieved this in a simple manner.

In order to determine whether the extra complexity of accounting for all choices was not problematic, we rescored a random sample of 20 items. For this rescoring, only the first four choices made by participants were counted and scored; 4 points were given to the first choice, 3 for the second, 2 for the third, and 1 for the fourth (equivalent to the first row of scoring in Table 1). This rescoring made a difference to 1 out of 20 items: $a d-$ vance (see Table 2). For advance, the rescoring resulted in a slight reduction of the normed score for several pictures (because 1 participant had chosen more than 4 pictures, so the points for these choices were removed). For the other items, all participants chose 4 pictures, so the rescoring made no difference. In the rare cases in which more than four pictures were chosen, the original scoring included this variability by increasing normed scores across pictures, as intended.

For each verb, the final scores for each picture were averaged across participants (the sum of all the points for that picture divided by the total number of participants). This allowed us to identify the pictures that were most preferred for each verb.

For each attribute, scores were summed across pictures with that attribute (e.g., horizontal or vertical axis, upward or downward arrow). For example, for the vertical axis, there were four pictures (two up and two down), whereas for upward motion, there were two pictures. The maximum score for one picture was 4 (i.e., if every participant ranked it as their first preference). This meant that summing across pictures produced scores above 4 for strongly selected attributes and below 1 or 2 for weakly selected attributes.

\section{Results and Discussion of Normed Scores}

Table 3 shows descriptive statistics for the normed scores for upward, downward, vertical, leftward, rightward, horizontal, toward (the circle/subject), and away (from the circle/subject) motion. The percentiles show the distribution of the items across normed scores. The mean values show that most items lack strong vertical motion (upward or downward), but the average value for horizontal motion is quite high (3.40), suggesting that horizontal pictures were used as a default option. The trade-off between horizontal and vertical motion produced a significant negative correlation $(n=299$, Pearson's $r=-.816, p<.001)$, lending weight to this idea. That is, those items with no strong vertical component (i.e., the majority of the items) have a horizontal arrow chosen by default (being a fairly neutral choice). For both upward and downward motion, the majority of items fall into the lower normed score bands between 0 and 2, reflecting the fact that only a subset of the 299 items (around 30 in each case) reflect events with strongly vertical motion (with normed values of 4 or more). The movement of entities in the vertical dimension and words that refer to that motion reflect the strong ecological

Table 2

Comparison of Original and Rescored Item Advance

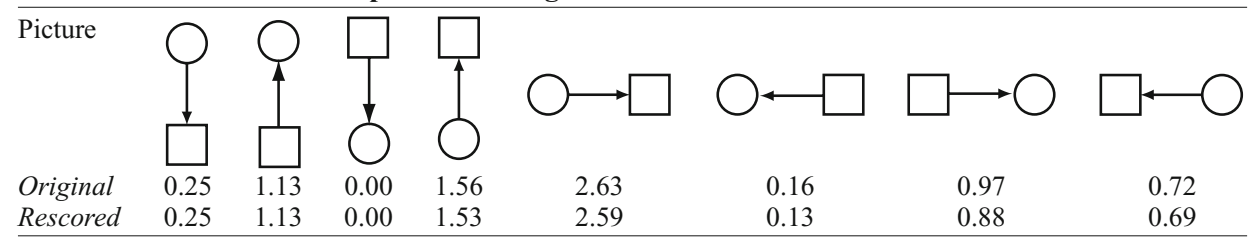

Note-Pictures are those used in the norming procedure and are included with the available data set. 
Table 3

Descriptive Statistics for Different Axes and Directions of Motion

From the Full Normed Set of 299 Verbs

\begin{tabular}{lcccccccc}
\hline \multirow{2}{*}{\begin{tabular}{c} 
Descriptive \\
\multicolumn{1}{c}{ Statistics }
\end{tabular}} & Up & Down & Vertical & Left & Right & Horizontal & Toward & Away \\
\cline { 2 - 9 } Mean & 1.68 & 1.82 & 3.50 & 1.41 & 1.99 & 3.40 & 2.30 & 4.60 \\
Median & 1.28 & 1.41 & 3.38 & 1.50 & 2.10 & 3.72 & 2.03 & 4.57 \\
Standard deviation & 1.38 & 1.37 & 1.27 & 0.80 & 1.08 & 1.73 & 1.74 & 1.83 \\
Variance & 1.91 & 1.87 & 1.61 & 0.64 & 1.16 & 3.01 & 3.05 & 3.36 \\
Range & 6.34 & 6.28 & 6.94 & 3.57 & 4.70 & 6.86 & 7.37 & 8.43 \\
Minimum & 0.00 & 0.00 & 0.14 & 0.00 & 0.00 & 0.00 & 0.00 & 0.16 \\
Maximum & 6.34 & 6.28 & 7.08 & 3.57 & 4.70 & 6.86 & 7.37 & 8.59 \\
Percentile & & & & & & & & \\
10 & 0.31 & 0.44 & 1.91 & 0.27 & 0.53 & 1.00 & 0.22 & 2.17 \\
20 & 0.60 & 0.80 & 2.37 & 0.58 & 1.00 & 1.58 & 0.59 & 3.06 \\
30 & 0.73 & 0.90 & 2.60 & 0.73 & 1.10 & 1.93 & 0.87 & 3.27 \\
40 & 0.84 & 0.97 & 2.83 & 0.87 & 1.33 & 2.33 & 1.03 & 3.50 \\
50 & 1.06 & 1.22 & 3.17 & 1.17 & 1.63 & 3.00 & 1.56 & 3.93 \\
60 & 1.28 & 1.41 & 3.38 & 1.50 & 2.10 & 3.72 & 2.03 & 4.57 \\
70 & 1.53 & 1.70 & 3.70 & 1.78 & 2.31 & 4.17 & 2.53 & 5.06 \\
80 & 1.93 & 2.03 & 4.03 & 1.97 & 2.57 & 4.66 & 3.25 & 5.70 \\
90 & 2.10 & 2.37 & 4.30 & 2.03 & 2.77 & 4.77 & 3.58 & 5.97 \\
\hline
\end{tabular}

Note-Percentiles are cutoff values for 10 equal groups.

constraint of gravity. Entities can move either with gravity (downward) or against it (upward). Thus, items with strong vertical motion typically are those that express motion of some entity in one particular direction, either with (e.g., drop, fall) or against (e.g., rise, ascend) gravity.

For horizontal motion, there is a reasonably flat distribution across values $1-4$, with a peak around the band $4-5$, again supporting the increased use of the horizontal axis as a common or default picture choice. It is not clear what leftward or rightward motion might reflect, because, in contrast to vertical motion, horizontal motion has no strong ecological constraint for a particular direction: Events should be equally likely to occur from left to right or right to left. The mean values show a slight tendency for higher rightward motion (i.e., left to right), which might reflect reading direction and the order of items in the rebus sentences, in which the agent (a circle) was always on the left and, for transitive sentences, the object (a square) was always on the right. We tried to reduce any perceptual bias this might have produced by using words instead of pictures in the target rebus sentence. However, due to the participant sample (native English speakers), the higher values for rightward motion may reflect a deeper spatial bias due to the reading direction of English (left to right). In a sentence-picture matching task, Italian participants, who read from left to right, were faster when the pictures showed the subject (agent) on the left. In contrast, Arab participants, who read from right to left, were faster at matching when the subject (agent) was on the right (Maass \& Russo, 2003; cf. Chan \& Bergen, 2005). If reading direction does bias participants to process pictured actions in the same direction, it is perhaps not surprising that our participants selected more pictures with left-to-right motion when selecting pictures to represent a sentence in English. Note also that a bias to process actions from left to right has been found; for example, when performing a sentence-picture matching task, participants respond more quickly to pictures that depict the agent on the left. This effect is independent of the particular sentence (i.e., regardless of whether the action proceeds from agent to patient, as in push, or from patient to agent, as in pull) (Chatterjee, Southwood, \& Basilico, 1999). Maass and Russo also found this facilitation for events that were depicted left to right in both Italian and Arab participants. These results are interpreted in terms of hemispheric specialization, with the left hemisphere directing attention from left to right and conceiving basic action schemata from left to right (Chatterjee, 2001). This throws up the interesting possibility that, if norms similar to those presented here were collected from populations who read from right to left, the results may show a similar tendency to select picture with the agent on the left (and therefore higher scores for rightward motion). However, there is some evidence that reading direction has a much stronger effect than the proposed event-schema bias, showing stronger influences in sentence-picture matching (Maass \& Russo, 2003).

For motion toward or away from the subject (the circle), when the verb in question is transitive, this probably reflects the direction of causality from subject to object (e.g., push) or from object to subject (e.g., pull). For motion toward the subject, the average mean is reasonably low (2.30), and items cluster around the lower end of the normed score bins (between 0 and 4), reflecting the relative rarity of items that express direction/causality toward the subject (e.g., inhale, pull, or take). For motion away from the subject, the mean value is high (4.60) and items are distributed normally around the central values (4 and 5), reflecting the fact that an arrow away from the observer can be used to represent agentive motion (walk, run, move) and causality away from the subject (push, hit, touch), therefore making them appropriate for many items in the set (similar to horizontal arrows). Given that pictures are two-dimensional, users of the norms are warned that the toward/away score should be interpreted with caution, 
Table 4

Up, Down, and Control Items Used in Meteyard, Bahrami, and Vigliocco (2007) and Meteyard, Zokaei, Bahrami, and Vigliocco (2008)

\begin{tabular}{|c|c|c|}
\hline$U p$ & Down & Control \\
\hline fly & dig & eat \\
\hline hop & rot & jog \\
\hline grow & bomb & burn \\
\hline jump & dive & drag \\
\hline leap & drip & kick \\
\hline lift & drop & pull \\
\hline rise & dump & quit \\
\hline soar & fall & race \\
\hline arise & hang & wash \\
\hline boost & pour & swim \\
\hline climb & $\sin \mathrm{k}$ & rust \\
\hline erect & crush & catch \\
\hline float & drain & chase \\
\hline hoist & lapse & cross \\
\hline raise & lower & eject \\
\hline stack & slump & enter \\
\hline surge & plunge & glide \\
\hline tower & tumble & sweep \\
\hline ascend & wither & attack \\
\hline emerge & cascade & depart \\
\hline expand & crumble & exhale \\
\hline launch & decline & reward \\
\hline spring & deflate & snatch \\
\hline sprout & depress & sneeze \\
\hline elevate & descend & tunnel \\
\hline inflate & plummet & stagger \\
\hline support & collapse & tremble \\
\hline escalate & decrease & distract \\
\hline heighten & demolish & exchange \\
\hline increase & deteriorate & retrieve \\
\hline
\end{tabular}

Note-Items are ordered according to number of letters (length) and alphabetically within a particular length.

because it may reflect differing aspects of an event (e.g., the movement of the subject, as in climb/bounce, or the direction of causality between the subject and object, as in distract/hope), depending on the particular item.

The advantage of having a relatively large item set is that, even for those parameters for which items are rare (e.g., strong vertical motion, strong motion/causality toward the subject), there still are items in the set that can be selected, as indicated by the maximum values for each motion component (all $>6$, except for left and right, both of which are $>3.5$ ). As a rule of thumb, values of 4 and above can be considered a strong normed score. This value is given to the first choice by a given participant; an average across subjects that approaches or exceeds 4 indicates that a majority of participants chose a picture with that quality (e.g., upward motion, horizontal motion) as a first choice. Values of greater than 4 reflect normed scores that are summed across pictures; for example, of the picture choices given to participants, two reflected upward motion (one with an arrow going toward the subject/circle and one with an arrow going away from the subject/circle), so the score for upward motion is produced by adding the normed score from these two pictures. For all axes and directions of motion summarized in the table, it is possible to find items with very high normed scores (e.g., above 6) and very low normed scores (around 0).

\section{Experimental Validation of the Normed Scores}

In previous experiments, we focused on the vertical axis: $U p$ and down words were selected by using items with a high normed score for upward or downward motion, respectively. We selected control words by using items that had no salient vertical motion - that is, with low or equal scores across different motion directions or low vertical motion scores. We selected 30 items for each word group (up, down, and control). The words selected were as concrete as possible, and could or did refer to concrete events (see Table 4). The control words were matched by group to the up and down words for frequency and length measures (all comparisons, $t<1.2$; see Table 5). Because the items were to be presented as auditory stimuli, the three word groups were matched for number of phonemes (all comparisons, $t<1$ ) and phonological neighborhood size (all comparisons, $t<1.2$ ) (Pisoni, Hernandez, \& Speech \& Hearing Lab, 2007). The up and down words were matched on all measures, except for CELEX combined frequency (Baayen, Piepenbrock, \& Gulikers, 1995), for which up words were slightly more frequent $[t(58)=$ $2.081, p<.05]$. These word groups were then used in a number of experiments (Meteyard, 2008), two of which have been published and are summarized here (Meteyard et al., 2007; Meteyard et al., 2008).

Table 5

Mean and Standard Deviation (SD) Values for Each Matched Variable

\begin{tabular}{|c|c|c|c|c|c|c|}
\hline \multirow[b]{4}{*}{ Lexical Variable } & \multicolumn{6}{|c|}{ Each Matched Variable } \\
\hline & \multicolumn{6}{|c|}{ Word Category } \\
\hline & \multicolumn{2}{|c|}{ Control } & \multicolumn{2}{|c|}{ Down } & \multicolumn{2}{|c|}{ Up } \\
\hline & $M$ & $S D$ & $M$ & $S D$ & $M$ & $S D$ \\
\hline \multicolumn{7}{|l|}{ Frequency } \\
\hline CELEX spoken & 4.07 & 8.62 & 3.17 & 7.69 & 5.60 & 9.68 \\
\hline CELEX combined & 6.27 & 10.49 & 3.63 & 6.58 & 10.17 & 15.88 \\
\hline \multicolumn{7}{|c|}{ Phonological neighborhood } \\
\hline Luce's $N$ & 2.63 & 5.14 & 1.77 & 6.72 & 2.24 & 4.40 \\
\hline \multicolumn{7}{|l|}{ Length } \\
\hline Letters & 5.23 & 1.41 & 5.63 & 1.85 & 5.37 & 1.38 \\
\hline Syllables & 1.37 & 0.49 & 1.60 & 0.86 & 1.43 & 0.63 \\
\hline Phonemes & 4.37 & 1.38 & 4.47 & 2.03 & 4.03 & 1.10 \\
\hline
\end{tabular}

Note-Matching is based on group means. 
In Meteyard et al. (2007), participants performed a standard motion-detection task (deciding whether random dot displays contained coherent or random motion), while passively comprehending aurally presented blocks of words (up, down, or control word blocks). Participants had two separate sessions that were identical except for the direction of coherent motion. In one session, motion was upward, and in the other, it was downward. The coherence of the motion in the target displays was set at each participant's individual threshold (making the motion signal ambiguous and the task difficult), which was established in a separate thresholding procedure before the main experiment.

There were six conditions, all within subjects (control words-upward motion, control words-downward motion, up words-upward motion, up words-downward motion, down words-upward motion, down words-downward motion). We collapsed these across motion direction to produce three conditions: control (control words-upward motion, control words-downward motion), match (up words-upward motion, down words-downward motion), and mismatch ( $u p$ words-downward motion, down wordsupward motion). Results showed that the $d^{\prime}$ for motion detection was significantly lower for the mismatch condition in comparison with both control and match conditions. That is, when the meaning of the words conflicted with the direction of motion to be detected, perceptual sensitivity was reduced. Because the words were blocked, this effect may have been due to participants' recognizing the meanings of the words (e.g., 30 consecutive $u p$ words create a strong directional context) and adopting a particular strategy or attentional set. However, participants' decision criterion (as measured by $c$ ) was significantly reduced for the match condition, suggesting that such strategic processes were at work in the match, as opposed to the mismatch, condition. That is, in the match condition, participants reduced the threshold at which they would accept a visual display as having coherent motion. Thus, there was a separation in the conditions at which word meaning affected perceptual $\left(d^{\prime}\right)$ and decision $(c)$ processes. Such a separation allowed us to argue for the presence of an interaction between language comprehension and visual perception that is more direct than one mediated strictly by attentional/strategic processes.

Our first experiment (Meteyard et al., 2007) demonstrated effects of language comprehension on low-level visual perception of motion; in a second experiment (Meteyard et al., 2008), we set out to assess, in a parallel fashion, whether low-level perception can affect word comprehension. We presented participants with a visual lexical decision task. Items were presented on screen for $1,500 \mathrm{msec}$, with a random dot display presented behind the word at onset for $200 \mathrm{msec}$. In a between-subjects manipulation, this random dot display was set at each participant's individual threshold level, at $30 \%, 60 \%$, or $90 \%$. We again collapsed results across motion directions to produce the control, match, and mismatch conditions. Results showed that, when the motion was at threshold and mismatched to the direction of the word, reaction times to make the lexical decision were longer. In line with previ- ous findings (e.g., Tsushima, Sasaki, \& Watanabe, 2006), we assume that, when the motion was above threshold (at $30 \%, 60 \%$, or $90 \%$ ), it was suppressed by executive processes, removing its ability to interfere with the lexical decision. The error rates also supported this conclusion, being higher for motion words (in both match and mismatch conditions) when motion was above threshold but not when motion was at threshold. This can be accounted for by assuming that the suppression of motion signals above threshold comes at a cost; hence, there is greater disruption for the processing of motion words, regardless of congruence. Thus, Meteyard et al. (2008) demonstrated that low-level vision processes affect single-word comprehension. The presence of a dissociation between conditions in which motion was at threshold and when it was not support the inference that these effects are not mediated by attentional processes.

The results supported a more direct connection between visual areas that process motion and the semantic processing of motion words. These two experiments validate the norming procedure and its ability to capture the salient motion content in given verbs. Crucially, both experiments presented single words, rather than embedding the verbs in sentences; thus, we assessed both the motion content carried by the verb alone and the ability of the norms to capture such content.

\section{Applications for the Normed Scores}

In this section, we present a number of questions that remain to be addressed and to which our norms could be fruitfully applied.

The first step in exploring interactions between language and perception/action is to establish a set of items that have sensory or motor content, such as words that refer to tangible objects (e.g., cat, ball, or words for any other concrete object) or to events (e.g., kicking, licking). For words that refer to concrete objects or events performed with particular effectors, it is self-evident which sensory or motor content should be present and therefore which sensory or motor systems are implicated (e.g., Barsalou, 1999). Events define relationships between entities and are temporally extended; therefore, words referring to events (verbs) are necessarily more abstract than are words referring to objects (nouns). This fact is highly relevant when dealing with motion events, because they can be made concrete or abstract by changing the participating entities. For example, compare The curtain rose with The price rose, the former describing a concrete motion event and the latter describing an abstract motion event in line with the concrete and abstract subject nouns. Although the verb to rise clearly refers to upward motion, it is not obvious how the salience of the motion changes with the noun that it instantiates. Our experiments so far (Meteyard et al., 2007; Meteyard et al., 2008) have shown that the norms reflect the motion content of single verbs; therefore, future experiments will be able to use different sentences to explore how that content interacts with other sentence items (i.e., subject and object nouns, prepositional and adverbial phrases); see Zwaan and Taylor (2006) for an elegant start to such work. Of particular interest is whether 
interactions between comprehension and perception are seen when sentences with the same verb are given concrete or abstract subjects. There are inconsistent results in the literature in cases where concreteness is manipulated (e.g., Bergen et al., 2007; Richardson et al., 2003). We expect that more consistent results will be found for concrete events and that the strength of interaction between perceptual processes and comprehension may be modulated by the concreteness of the objects in the described event.

In the experiments we have completed so far (Meteyard et al., 2007; Meteyard et al., 2008; and the present study), we have focused on the vertical axis of motion, using strongly "upward" and "downward" verbs. The norms also allow verbs in the horizontal dimension to be extracted. As mentioned above, the horizontal dimension of actions has been shown to be influenced by reading direction (Chan \& Bergen, 2005; Maass \& Russo, 2003), but there is also a proposed language-independent bias to conceptualize events as unfolding from left to right (Chatterjee, 2001; Chatterjee et al., 1999). The norms presented here could be analyzed to determine whether higher scores for rightward motion are present across all items with high horizontal scores or whether it changes relative to particular qualities of the item. For example, abstract events may be more vulnerable to a left-to-right bias, whether from reading direction or from a more basic conceptual bias (because there is no perceptual referent to constrain judgments). There are a few items with higher scores for rightto-left than for left-to-right motion (e.g., return, recede); the qualities of these items may be of interest to researchers who are interested in spatial biases for actions (e.g., do they all imply an action with outward and return motion?). If translation equivalents can be found for verbs in this set with strong horizontal scores, it would be interesting to have similar norms collected from populations who read from right to left. The relative bias from reading direction may be reflected in normed scores, but it is also possible that a language-independent bias may be evidenced (because the task encourages participants to think about the event rather than the words). These suggestions all come with caveats: that participants who normed these items are likely to have a rightward motion bias, in line with the English reading direction, and that the task requires the reading of rebus sentences.

When different visual processes (e.g., visuospatial attention and motion perception) are combined with comprehension, they appear to be affected in different ways. For example, for visuospatial attention, detection of a target object is slower when the semantic and perceptual stimuli are congruent (e.g., a sentence referring to upward motion and an object at the top of the visual field; Bergen et al., 2007; Richardson et al., 2003). In contrast, pictures that approximate the motion of an object toward or away from the reader are judged more quickly when paired with sentences that describe congruent motion (Zwaan et al., 2004). The perception of dynamic motion has been shown to be worse when paired with incongruent motion words (Meteyard et al., 2007), and lexical decision on motion words is slower when incongruent motion patterns are displayed concurrently with the words (Meteyard et al.,
2008). Conversely, sensibility or grammaticality judgments of sentences describing motion were found to be slower when paired with the perception of congruent dynamic motion (Kaschak et al., 2005; Kaschak et al., 2006). There is clearly much work to be done in order to bring together these different findings and to explore where and how such interactions take place; we hope that by our making these norms available, consistent item sets can be developed and tested. Item-specific effects can then be ruled out as an explanation for these divergent results.

The brief summary above has shown how research into eye movement also provides support for the role of perceptual and motor information in semantic representations. Researchers who are interested in how single-word and sentence comprehension interact with eye movements (e.g., during the tracking or identification of visual targets) may find the norms useful for selecting lexical stimuli. It would be interesting to investigate whether items with higher normed scores for motion interfere to a greater extent with eye movements when tracking objects moving on a screen, in contrast to items with lower or less consistent normed scores. The normed scores should reflect the relative salience of motion for a particular event; it would be interesting to know whether this relative salience is reflected in effects on eye movements or on other perceptual tasks.

\section{Conclusions}

We have presented norms for the axis and direction of motion in 299 English verbs. The primary advantage of this data set is its size, which allows flexibility in item selection, while controlling for important parameters, such as frequency, length, and orthographic (or phonological) variables. We have demonstrated this in two experiments that used items from this data set to make up three conditions (i.e., up, down, control) of 30 verbs that were reasonably well matched. These two experiments validated the ability of the norming procedure (adapted from Richardson et al., 2001) to capture the motion content of single words. The number of items in the set also indicates that the range of scores is large, again allowing considerable flexibility in item selection across a number of motion directions. We hope that these norms will be useful for all researchers interested in the semantic representation of motion and particularly for investigations that, in order to uncover the precise nature of interactions between language and sensory-motor processes, explore predictions from embodied theories of semantic content.

The norms in the main set are provided for each individual picture (columns 3-10) and are summed across pictures to give upward, downward, vertical, leftward, rightward, horizontal, toward, and away motion scores (columns 11-18). Normed values are provided in tab-delimited text files that can be used easily in Excel or other spreadsheet software. Columns 3-10 are labeled with the picture to which those values refer, and pdf files of these pictures are provided with the corresponding file name. Columns 11-18 are labeled with the relevant motion direction (e.g., upward, leftward). Pdf documents of the norming materials and instructions, and a text document containing details of the materials, are also provided. 


\section{AUTHOR NOTE}

This work was supported by an ESRC studentship (PTA-030-200300379) to L.M. We thank Matthew Whalley for help on the revised version of the manuscript. Address correspondence to L. Meteyard, MRC Cognitive and Brain Science Unit, 15 Chaucer Road, Cambridge CB2 7EF, England (e-mail: meteyard@gmail.com).

\section{REFERENCES}

BaAyen, R. H., Piepenbrock, R., \& Gulikers, L. (1995). The CELEX lexical database [CD-ROM]. Philadelphia: University of Pennsylvania, Linguistic Data Consortium.

Barsalou, L. W. (1999). Perceptual symbol systems. Behavioral \& Brain Sciences, 22, 577-609. doi:10.1017/S0140525X9900214

Bergen, B. K., Lindsay, S., Matlock, T., \& Narayanan, S. (2007). Spatial and linguistic aspects of visual imagery in sentence comprehension. Cognitive Science, 31, 733-764. doi:10.1080/03640210701530748

Chambers, C. G., Tanenhaus, M. K., Eberhard, K. M., Filip, H., \& Carlson, G. N. (2002). Circumscribing referential domains during real-time language comprehension. Journal of Memory \& Language, 47, 30-49. doi:10.1006/jmla.2001.2832

Chambers, C. G., Tanenhaus, M. K., \& Magnuson, J. S. (2004). Actions and affordances in syntactic ambiguity resolution. Journal of Experimental Psychology: Learning, Memory, \& Cognition, 30, 687696. doi:10.1037/0278-7393.30.3.687

Chan, T. T., \& Bergen, B. (2005). Writing direction influences spatial cognition. Proceedings of the 27th Annual Conference of the Cognitive Science Society. In B. G. Bara, L. Barsalou, \& M. Bucciarelli (Eds.), (pp. 412-417). Mahwah, NJ: Erlbaum.

Chatterjee, A. (2001). Language and space: Some interactions. Trends in Cognitive Sciences, 5, 55-61. doi:10.1016/S1364 $-6613(00) 01598-9$

Chatterjee, A., Southwood, M. H., \& Basilico, D. (1999). Verbs, events and spatial representations. Neuropsychologia, 37, 395-402. doi:10.1016/S0028-3932(98)00108-0

Dahan, D., \& Tanenhaus, M. K. (2005). Looking at the rope when looking for the snake: Conceptually mediated eye movements during spoken-word recognition. Psychonomic Bulletin \& Review, 12, 453-459.

Estes, Z., Verges, M., \& Barsalou, L. W. (2008). Head up, foot down: Object words orient attention to the object's typical location. Psychological Science, 19, 93-97. doi:10.1111/j.1467-9280.2008.02051.x

FoDOR, J. A. (1987). Psychosemantics: The problem of meaning in the philosophy of mind. Cambridge, MA: MIT Press.

Henderson, J. M., \& Ferreira, F. (EDS.) (2004). The interface of language, vision, and action: Eye movements and the visual world. New York: Psychology Press.

Kaschak, M. P., Madden, C. J., Therriault, D. J., Yaxley, R. H., Aveyard, M. E., Blanchard, A. A., \& ZwaAn, R. A. (2005). Perception of motion affects language processing. Cognition, 94, B79-B99. doi:10.1016/j.cognition.2004.06.005

Kaschak, M. P., Zwan, R. A., Aveyard, M., \& Yaxley, R. H. (2006). Perception of auditory motion affects language processing. Cognitive Science, 30, 733-744. doi:10.1207/s15516709 $\operatorname{cog} 0000 \_54$

KossLYN, S. M. (1996). Image and brain: The resolution of the imagery debate. Cambridge, MA: MIT Press.

Laeng, B., \& TeODorescu, D.-S. (2002). Eye scanpaths during visual imagery reenact those of perception of the same visual scene. Cognitive Science, 26, 207-231.

LAKOFF, G. (1987). Women, fire, and dangerous things: What categories reveal about the mind. Chicago: University of Chicago Press.

LEvIN, B. (1993). English verb classes and alternations: A preliminary investigation. Chicago: University of Chicago Press.

MaAss, A., \& Russo, A. (2003). Directional bias in the mental representation of spatial events: Nature or culture? Psychological Science, 14, 296-301. doi:10.1111/1467-9280.14421

MatLOCK, T., \& RichaRdSON, D. C. (2004). Do eye movements go with fictive motion? Proceedings of the 26th Annual Conference of the Cognitive Science Society. Mahwah, NJ: Erlbaum.
Meteyard, L. (2008). Motion seen and understood: Interactions between language comprehension and visual perception. Unpublished doctoral dissertation, University of London. Available at www .meteyard.com/academic.

Meteyard, L., Bahrami, B., \& Vigliocco, G. (2007). Motion detection and motion verbs: Language affects low-level visual perception. Psychological Science, 18, 1007-1013. doi:10.1111/j.1467 $-9280.2007 .02016 . x$

Meteyard, L., Zokaei, N., Bahrami, B., \& Vigliocco, G. (2008). Visual motion interferes with lexical decision on motion words. Current Biology, 18, R732-R734. doi:10.1016/j.cub.2008.07.016

MurPHY, G. L. (2002). The big book of concepts. Cambridge, MA: MIT Press.

Myung, J., Blumstein, S. E., \& Sedivy, J. C. (2005). Playing on the typewriter, typing on the piano: Manipulating knowledge of objects. Cognition, 98, 223-243. doi:10.1016/j.cognition.2004.11.010

Perky, C. W. (1910). An experimental study of imagination. American Journal of Psychology, 21, 422-452. doi:10.2307/1413350

Pisoni, D., Hernandez, L., \& SPeech \& Hearing Lab (2007). Speech $\&$ hearing lab neighborhood database. Retrieved January 1, 2005, from http://128.252.27.56/Neighborhood/Home.asp.

Pulvermüller, F. (2001). Brain reflections of words and their meaning. Trends in Cognitive Sciences, 5, 517-524. doi:10.1016/S1364 -6613(00)01803-9

Pylyshyn, Z. W. (1985). Computation and cognition: Toward a foundation for cognitive science. Cambridge, MA: MIT Press.

Richardson, D. C., Spivey, M. J., Barsalou, L. W., \& McRae, K. (2003). Spatial representations activated during real-time comprehension of verbs. Cognitive Science, 27, 767-780.

Richardson, D. C., Spivey, M. J., Edelman, S., \& Naples, A. J. (2001). "Language is spatial": Experimental evidence for image schemas of concrete and abstract verbs. Proceedings of the 23rd Annual Conference of the Cognitive Science Society. Mahwah, NJ: Erlbaum.

Spivey, M. J., \& GeNG, J. J. (2001). Oculomotor mechanisms activated by imagery and memory: Eye movements to absent objects. Psychological Research, 65, 235-241. doi:10.1007/s004260100059

Spivey, M. J., Richardson, D. C., Tyler, M. J., \& Young, E. E. (2000). Eye movements during comprehension of spoken scene descriptions. In L. R. Gleitman \& A. K. Joshi (Eds.). Proceedings of the 22nd Annual Conference of the Cognitive Science Society (pp. 487-492). Mahwah, NJ: Erlbaum.

Stanfield, R. A., \& ZwaAn, R. A. (2001). The effect of implied orientation derived from verbal context on picture recognition. Psychological Science, 12, 153-156. doi:10.1111/1467-9280.00326

Tsushima, Y., Sasaki, Y., \& Watanabe, T. (2006). Greater disruption due to failure of inhibitory control on an ambiguous distractor. Science, 314, 1786-1788. doi:10.1126/science. 1133197

ZwaAn, R. A. (1996). Processing narrative time shifts. Journal of Experimental Psychology: Learning, Memory, \& Cognition, 22, 11961207. doi:10.1037/0278-7393.22.5.1196

Zwaan, R. A., Madden, C. J., Yaxley, R. H., \& Aveyard, M. E. (2004). Moving words: Dynamic representations in language comprehension. Cognitive Science, 28, 611-619.

Zwaan, R. A., Stanfield, R. A., \& Yaxley, R. H. (2002). Language comprehenders mentally represent the shape of objects. Psychological Science, 13, 168-171. doi:10.1111/1467-9280.00430

ZwaAn, R. A., \& TAYlor, L. J. (2006). Seeing, acting, understanding: Motor resonance in language comprehension. Journal of Experimental Psychology: General, 135, 1-11. doi:10.1037/0096-3445.135.1.1

\section{SUPPLEMENTAL MATERIALS}

The word norms and picture stimuli from this article may be downloaded from brm.psychonomic-journals.org/content/supplemental.

(Manuscript received August 21, 2008; revision accepted for publication December 30, 2008.) 